\title{
Theoretical aspects of electroweak symmetry breaking in SUSY models
}

\author{
Roman Nevzorov ${ }^{* \dagger}$ \\ University of Hawaii \\ E-mail: nevzorov@phys.hawaii.edu
}

The electroweak (EW) symmetry breaking in the simplest supersymmetric (SUSY) extensions of the standard model (SM), i.e. minimal and next-to-minimal supersymmetric standard models (MSSM and NMSSM), is considered. The spectrum of the Higgs particles, upper bound on the mass of the lightest Higgs boson and little hierarchy problem are discussed. The breakdown of gauge symmetry and Higgs phenomenology within the $E_{6}$ inspired SUSY models with extra $U(1)^{\prime}$ factor are briefly reviewed.

The XIXth International Workshop on High Energy Physics and Quantum Field Theory 8-15 September 2010

Golitsyno, Moscow, Russia

* Speaker.

${ }^{\dagger}$ On leave of absence from the Theory Department, ITEP, Moscow, Russia. 


\section{Introduction}

Nowadays, there are serious reasons to believe that new particles and interactions should exist at the TeV scale. We expect that LHC might be able to shed light on the physics beyond the Standard Model (SM), origin of dark matter and the mechanism of electroweak (EW) symmetry breaking in the near future. At the moment the supersymmetric (SUSY) models are the best motivated extensions of the SM. SUSY models are defined by the field content, structure of gauge interactions and superpotential. In order to give both up- and down-type fermions a mass and to ensure anomaly cancellation the Higgs sector of the minimal supersymmetric standard model (MSSM) includes two Higgs doublets $\left(H_{1}\right.$ and $\left.H_{2}\right)$ with the opposite hypercharge. Also in SUSY models Rparity is normally imposed $\left(R=(-1)^{3(B-L)+2 S}\right)$. It forbids the baryon and lepton number violating operators that lead to rapid proton decay. R-parity ensures that the lightest supersymmetric particle (LSP) is absolutely stable and can play the role of dark matter. The most general renormalizable superpotential of the MSSM can be presented in the following form

$$
W_{M S S M}=\varepsilon_{i j}\left(y_{a b}^{U} Q_{a}^{j} u_{b}^{c} H_{2}^{i}+y_{a b}^{D} Q_{a}^{j} d_{b}^{c} H_{1}^{i}+y_{a b}^{L} L_{a}^{j} e_{b}^{c} H_{1}^{i}+\mu H_{1}^{i} H_{2}^{j}\right),
$$

where $i, j=1,2,3$ are the $S U(2)$ and $a, b=1,2,3$ are the generation indices. In Eq. (1.1) colour indices are suppressed.

Since SUSY particles are considerably heavier than observed quarks and leptons supersymmetry must be broken. Thus the Lagrangian of SUSY models can be written as

$$
\mathscr{L}=\mathscr{L}_{\text {SUSY }}+\mathscr{L}_{\text {soft }}
$$

where $\mathscr{L}_{\text {soft }}$ contains soft SUSY breaking terms that break supersymmetry but do not induce quadratic divergences that get cancelled automatically within the models based on the exact global supersymmetry. In the MSSM the set of the soft SUSY breaking terms includes

$$
\begin{aligned}
-\mathscr{L}_{\text {soft }} & =\sum_{i} m_{i}^{2}\left|\varphi_{i}\right|^{2}+\left(\frac{1}{2} \sum_{\alpha} M_{\alpha} \tilde{\lambda}_{\alpha} \tilde{\lambda}_{\alpha}+\sum_{a, b}\left[A_{a b}^{U} y_{a b}^{U} \tilde{Q}_{a} \tilde{u}_{b}^{c} H_{2}\right.\right. \\
& \left.\left.+A_{a b}^{D} y_{a b}^{D} \tilde{Q}_{a} \tilde{d}_{b}^{c} H_{1}+A_{a b}^{L} y_{a b}^{L} \tilde{L}_{a} \tilde{e}_{b}^{c} H_{1}\right]+B \mu H_{1} H_{2}+\text { h.c. }\right),
\end{aligned}
$$

where $\varphi_{i}$ are scalar components of chiral superfields while $\tilde{\lambda}_{\alpha}$ are gaugino fields. To avoid finetuning SUSY breaking mass parameters are expected to be in the TeV range.

\section{EW symmetry breaking in the MSSM}

Including soft SUSY breaking terms and radiative corrections, the Higgs effective potential in the MSSM can be written as

$$
\begin{gathered}
V=m_{1}^{2}\left|H_{1}\right|^{2}+m_{2}^{2}\left|H_{2}\right|^{2}-m_{3}^{2}\left(H_{1} H_{2}+h . c .\right)+\frac{g_{2}^{2}}{8}\left(H_{1}^{+} \sigma_{a} H_{1}+H_{2}^{+} \sigma_{a} H_{2}\right)^{2} \\
+\frac{g^{\prime 2}}{8}\left(\left|H_{1}\right|^{2}-\left|H_{2}\right|^{2}\right)^{2}+\Delta V
\end{gathered}
$$

where $g^{\prime}=\sqrt{3 / 5} g_{1}, g_{2}$ and $g_{1}$ are the low energy (GUT normalised) $S U(2)_{W}$ and $U(1)_{Y}$ gauge couplings, $m_{1}^{2}=m_{H_{1}}^{2}+\mu^{2}, m_{2}^{2}=m_{H_{2}}^{2}+\mu^{2}$ and $m_{3}^{2}=-B \mu$. In Eq. (2.1) $\Delta V$ represents the contribution of loop corrections to the Higgs effective potential. 
At the physical minimum of the scalar potential (2.1) the Higgs fields develop vacuum expectation values (VEVs)

$$
<H_{1}>=\frac{1}{\sqrt{2}}\left(\begin{array}{c}
v_{1} \\
0
\end{array}\right), \quad<H_{2}>=\frac{1}{\sqrt{2}}\left(\begin{array}{c}
0 \\
v_{2}
\end{array}\right) .
$$

breaking the $S U(2)_{W} \times U(1)_{Y}$ gauge symmetry to $U(1)_{e m}$ associated with electromagnetism and generating the masses of all bosons and fermions. The value of $v=\sqrt{v_{1}^{2}+v_{2}^{2}} \simeq 246 \mathrm{GeV}$ is fixed by the Fermi scale. At the same time the ratio of the Higgs VEVs remains arbitrary. Hence it is convenient to introduce $\tan \beta=v_{2} / v_{1}$.

The vacuum configuration (2.2) is not the most general one. Because of the $S U(2)$ invariance of the Higgs potential (2.1) one can always make $\left\langle\mathrm{H}_{2}^{+}\right\rangle=0$ by virtue of a suitable gauge rotation. Then the requirement $\left\langle H_{1}^{-}\right\rangle=0$, which is a necessary condition to preserve $U(1)_{e m}$ in the physical vacuum, is equivalent to requiring the squared mass of the physical charged scalar to be positive. It imposes additional constraints on the parameter space.

At tree-level $\Delta V=0$ and the Higgs potential is described by the sum of the first five terms in Eq. (2.1). Notice that the Higgs self-interaction couplings in Eq. (2.1) are fixed and determined by the gauge couplings in contrast with the SM. At the tree-level the MSSM Higgs potential contains only three independent parameters: $m_{1}^{2}, m_{2}^{2}, m_{3}^{2}$. The stable vacuum of the scalar potential (2.1) exists only if

$$
m_{1}^{2}+m_{2}^{2}>2\left|m_{3}\right|^{2}
$$

This can be easily understood if one notice that in the limit $v_{1}^{2}=v_{2}^{2}$ the quartic terms in the Higgs potential vanish. In the considered case the scalar potential (2.1) remains positive definite only if the condition (2.3) is satisfied. Otherwise physical vacuum becomes unstable. On the other hand Higgs doublets acquire non-zero VEVs only when

$$
m_{1}^{2} m_{2}^{2}<\left|m_{3}\right|^{4} .
$$

Indeed, if $m_{1}^{2} m_{2}^{2}>\left|m_{3}\right|^{4}$ then all Higgs fields have positive masses for $v_{1}=v_{2}=0$ and the breakdown of EW symmetry does not take place. The conditions (2.3) and (2.4) also follow from the equations for the extrema of the Higgs boson potential. At tree-level the minimization conditions in the directions (2.2) in field space read:

$$
\begin{aligned}
& \frac{\partial V}{\partial v_{1}}=\left(m_{1}^{2}+\frac{\bar{g}^{2}}{8}\left(v_{1}^{2}-v_{2}^{2}\right)\right) v_{1}-m_{3}^{2} v_{2}=0, \\
& \frac{\partial V}{\partial v_{2}}=\left(m_{2}^{2}+\frac{\bar{g}^{2}}{8}\left(v_{2}^{2}-v_{1}^{2}\right)\right) v_{2}-m_{3}^{2} v_{1}=0
\end{aligned}
$$

where $\bar{g}=\sqrt{g_{2}^{2}+g^{\prime 2}}$. Solution of the minimization conditions (2.5) can be written in the following form

$$
\sin 2 \beta=\frac{2 m_{3}^{2}}{m_{1}^{2}+m_{2}^{2}}, \quad \frac{\bar{g}^{2}}{4} v^{2}=\frac{2\left(m_{1}^{2}-m_{2}^{2} \tan ^{2} \beta\right)}{\tan ^{2} \beta-1} .
$$

Requiring that $v^{2}>0$ and $|\sin 2 \beta|<1$ one can reproduce conditions (2.3) and (2.4). 
From Eqs. (2.3)-(2.6) it is easy to see that the appropriate breakdown of the EW symmetry can not be always achieved. In particular the breakdown of $S U(2)_{W} \times U(1)_{Y} \rightarrow U(1)_{e m}$ does not take place when $m_{2}^{2}=m_{1}^{2}$. This is exactly what happens in the constrained version of the $\operatorname{MSSM}(\mathrm{cMSSM})$ which implies that $m_{i}^{2}\left(M_{X}\right)=m_{0}^{2}, A_{a b}^{k}\left(M_{X}\right)=A, M_{\alpha}\left(M_{X}\right)=M_{1 / 2}$. Thus in the cMSSM $m_{2}^{2}\left(M_{X}\right)=m_{1}^{2}\left(M_{X}\right)$. Nevertheless the correct pattern of the EW symmetry breaking can be achieved within this SUSY model. Since the top-quark Yukawa coupling is large it affects the renormalisation group (RG) flow of $m_{2}^{2}(Q)$ rather strongly resulting in small or even negative values of $m_{2}^{2}(Q)$ at low energies that triggers the breakdown of the EW symmetry. This is the so-called radiative mechanism of the EW symmetry breaking (EWSB).

The radiative EWSB demonstrates the importance of loop effects in the considered process. In addition to the RG flow of all couplings one has to take into account loop corrections to the Higgs effective potential which are associated with the last term $\Delta V$ in Eq. (2.1). In the simplest SUSY extensions of the SM the dominant contribution to $\Delta V$ comes from the loops involving the top-quark and its superpartners because of their large Yukawa coupling $h_{t}$. In the one-loop approximation the contribution of the top-quark and its superpartners to $\Delta V$ is determined by the masses of the corresponding bosonic and fermionic states, i.e.

$$
\begin{gathered}
\Delta V=\frac{3}{32 \pi^{2}}\left[m_{\tilde{t}_{1}}^{4}\left(\ln \frac{m_{\tilde{t}_{1}}^{2}}{Q^{2}}-\frac{3}{2}\right)+m_{\tilde{t}_{2}}^{4}\left(\ln \frac{m_{\tilde{t}_{2}}^{2}}{Q^{2}}-\frac{3}{2}\right)-2 m_{t}^{4}\left(\ln \frac{m_{t}^{2}}{Q^{2}}-\frac{3}{2}\right)\right], \\
m_{\tilde{t}_{1,2}}=\frac{1}{2}\left(m_{Q}^{2}+m_{U}^{2}+2 m_{t}^{2} \pm \sqrt{\left(m_{Q}^{2}-m_{U}^{2}\right)^{2}+4 m_{t}^{2} X_{t}^{2}}\right)
\end{gathered}
$$

where $X_{t}=A_{t}-\mu / \tan \beta$ is a stop mixing parameter, $A_{t}$ is a trilinear scalar coupling associated with the top quark Yukawa coupling and $m_{t}$ is the running top quark mass

$$
m_{t}\left(M_{t}\right)=\frac{h_{t}\left(M_{t}\right)}{\sqrt{2}} v \sin \beta
$$

Initially the sector of EWSB involves eight degrees of freedom. However three of them are massless Goldstone modes which are swallowed by the $W^{ \pm}$and $Z$ gauge bosons. The $W^{ \pm}$and $Z$ bosons gain masses via the interaction with the neutral components of the Higgs doublets so that

$$
M_{W}=\frac{g_{2}}{2} v, \quad M_{Z}=\frac{\bar{g}}{2} v .
$$

When CP in the MSSM Higgs sector is conserved the remaining five physical degrees of freedom form two charged, one CP-odd and two CP-even Higgs states. The masses of the charged and CP-odd Higgs bosons are

$$
m_{A}^{2}=m_{1}^{2}+m_{2}^{2}+\Delta_{A}, \quad M_{H^{ \pm}}^{2}=m_{A}^{2}+M_{W}^{2}+\Delta_{ \pm},
$$

where $\Delta_{ \pm}$and $\Delta_{A}$ are the loop corrections. The CP-even states are mixed and form a $2 \times 2$ mass matrix. It is convenient to introduce a new field space basis $(h, H)$ rotated by the angle $\beta$ with respect to the initial one:

$$
\begin{aligned}
& \operatorname{Re} H_{1}^{0}=\left(h \cos \beta-H \sin \beta+v_{1}\right) / \sqrt{2}, \\
& \operatorname{Re} H_{2}^{0}=\left(h \sin \beta+H \cos \beta+v_{2}\right) / \sqrt{2} .
\end{aligned}
$$


In this new basis the mass matrix of the Higgs scalars takes the form [1]

$$
\begin{gathered}
M^{2}=\left(\begin{array}{cc}
M_{11}^{2} & M_{12}^{2} \\
M_{21}^{2} & M_{22}^{2}
\end{array}\right)=\left(\begin{array}{cc}
\frac{\partial^{2} V}{\partial v^{2}} & \frac{1}{v} \frac{\partial^{2} V}{\partial v \partial \beta} \\
\frac{1}{v} \frac{\partial^{2} V}{\partial v \partial \beta} & \frac{1}{v^{2}} \frac{\partial^{2} V}{\partial \beta^{2}}
\end{array}\right) \\
M_{22}^{2}=m_{A}^{2}+M_{Z}^{2} \sin ^{2} 2 \beta+\Delta_{22}, \quad M_{12}^{2}=M_{21}^{2}=-\frac{1}{2} M_{Z}^{2} \sin 4 \beta+\Delta_{12}, \\
M_{11}^{2}=M_{Z}^{2} \cos ^{2} 2 \beta+\Delta_{11},
\end{gathered}
$$

where $\Delta_{i j}$ represent the contributions from loop corrections. In Eqs. (2.11) the equations for the extrema of the Higgs boson effective potential are used to eliminate $m_{1}^{2}$ and $m_{2}^{2}$.

From Eqs. 2.8 and (2.11) one can see that at tree-level the masses and couplings of the Higgs bosons in the MSSM can be parametrised in terms $m_{A}$ and $\tan \beta$ only. The masses of the two $\mathrm{CP}-$ even eigenstates obtained by diagonalizing the matrix (2.10)-2.11) are given by

$$
m_{h_{1}, h_{2}}^{2}=\frac{1}{2}\left(M_{11}^{2}+M_{22}^{2} \mp \sqrt{\left(M_{22}^{2}-M_{11}^{2}\right)^{2}+4 M_{12}^{4}}\right) .
$$

The qualitative pattern of the Higgs spectrum depends very strongly on the mass $m_{A}$ of the pseudoscalar Higgs boson. With increasing $m_{A}$ the masses of all the Higgs particles grow. At very large values of $m_{A}\left(m_{A}^{2} \gg v^{2}\right)$, the lightest Higgs boson mass approaches its theoretical upper limit $\sqrt{M_{11}^{2}}$. Thus the top-left entry of the CP-even mass matrix (2.10)-(2.11) represents an upper bound on the lightest Higgs boson mass-squared. In the leading two-loop approximation the mass of the lightest Higgs boson in the MSSM does not exceed $130-135 \mathrm{GeV}$. This is one of the most important predictions of the minimal SUSY model that can be tested at the LHC in the near future.

It is important to study the spectrum of the Higgs bosons together with their couplings to the gauge bosons because LEP set stringent limits on the masses and couplings of the Higgs states. In the rotated field basis $(h, H)$ the trilinear part of the Lagrangian, which determines the interactions of the neutral Higgs states with the $Z$-boson, is simplified:

$$
L_{A Z H}=\frac{\bar{g}}{2} M_{Z} Z_{\mu} Z_{1 \mu} h+\frac{\bar{g}}{2} Z_{\mu}\left[H\left(\partial_{\mu} A\right)-\left(\partial_{\mu} H\right) A\right] .
$$

In this basis the SM-like CP-even component $h$ couples to a pair of $Z$ bosons, while the other one $H$ interacts with the pseudoscalar $A$ and $Z$. The coupling of $h$ to the $Z$ pair is exactly the same as in the SM. The couplings of the Higgs mass eigenstates to a $Z$ pair $\left(g_{Z Z h_{i}}, i=1,2,3\right)$ and to the Higgs pseudoscalar and $Z$ boson $\left(g_{Z A h_{i}}\right)$ appear because of the mixing between $h$ and $H$.

Following the traditional notations, one can define the normalised $R$-couplings as: $g_{V V h_{i}}=$ $R_{V V h_{i}} \times$ SM coupling $\left(V=Z, W^{ \pm}\right) ; g_{Z A h_{i}}=\frac{\bar{g}}{2} R_{Z A h_{i}}$. The absolute values of all these $R$-couplings vary from zero to unity. The relative couplings $R_{Z Z h_{i}}$ and $R_{Z A h_{i}}$ are given in terms of the angles $\alpha$ and $\beta$ :

$$
R_{V V h_{1}}=-R_{Z A h_{2}}=\sin (\beta-\alpha), \quad R_{V V h_{2}}=R_{Z A h_{1}}=\cos (\beta-\alpha),
$$



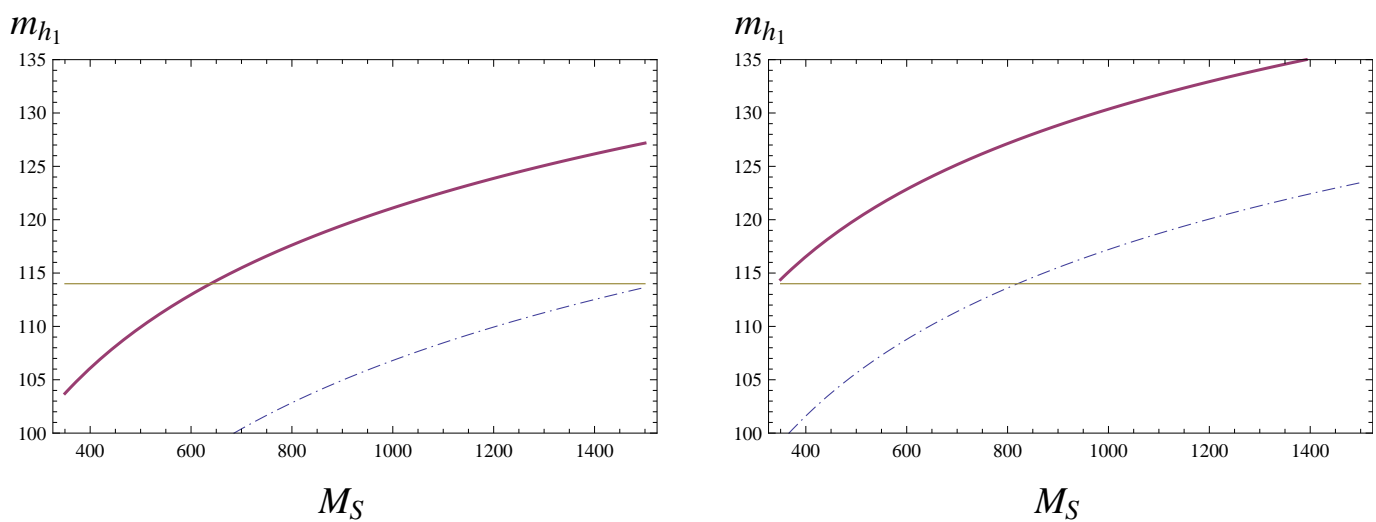

Figure 1: Left: The dependence of the one-loop lightest Higgs boson mass on $M_{S}$ for $\tan \beta=2$. Right: The one-loop mass of the lightest CP-even Higgs state versus $M_{S}$ for $\tan \beta=3$. The solid and dashed-dotted lines correspond to $X_{t}=2 M_{S}$ and $X_{t}=M_{S}$ respectively. The horizontal line represents the current LEP limit.

where the angle $\alpha$ is defined as follows:

$$
\begin{aligned}
& h_{1}=-\left(\sqrt{2} \operatorname{Re} H_{1}^{0}-v_{1}\right) \sin \alpha+\left(\sqrt{2} \operatorname{Re} H_{2}^{0}-v_{2}\right) \cos \alpha, \\
& h_{2}=\left(\sqrt{2} \operatorname{Re} H_{1}^{0}-v_{1}\right) \cos \alpha+\left(\sqrt{2} \operatorname{Re} H_{2}^{0}-v_{2}\right) \sin \alpha .
\end{aligned}
$$

From Eqs. (2.14) it becomes clear that in the MSSM the couplings of the lightest Higgs boson to the $\mathrm{Z}$ pair can be substantially smaller than in the SM. Therefore the experimental lower bound on the lightest Higgs mass in the MSSM is weaker than in the SM. On the other hand, when $m_{A}^{2} \gg v^{2}$ the lightest $\mathrm{CP}$-even Higgs state is predominantly the SM-like superposition of the neutral components of Higgs doublets $h$ so that $R_{Z Z h_{1}} \simeq 1$. In this case the lightest Higgs scalar has to satisfy LEP constraint on the mass of the SM-like Higgs boson, i.e. it should be heavier than $114.4 \mathrm{GeV}$.

Recent studies indicate that in the MSSM the scenarios with the light Higgs pseudoscalar $\left(m_{A} \sim 100 \mathrm{GeV}\right)$ are almost ruled out by LEP. Since $m_{A}$ tend to be large the SM-like Higgs boson must be relatively heavy. At the same time the lightest Higgs boson mass in the MSSM does not exceed $M_{Z} \simeq 91 \mathrm{GeV}$ at the tree-level. As a consequence in order to satisfy LEP constraints large contribution of loop corrections to the mass of the lightest CP-even Higgs state is required. When SUSY breaking scale $M_{S}$ is considerably larger than $M_{Z}$ and $m_{Q}^{2} \simeq m_{U}^{2} \simeq M_{S}^{2}$ the contribution of the one-loop corrections to $m_{h_{1}}^{2}$ in the leading approximation can be written as

$$
\Delta_{11}^{(1)} \simeq \frac{3 M_{t}^{4}}{2 \pi^{2} v^{2}}\left[\frac{X_{t}^{2}}{M_{S}^{2}}\left(1-\frac{1}{12} \frac{X_{t}^{2}}{M_{S}^{2}}\right)+\ln \left(\frac{M_{S}^{2}}{m_{t}^{2}}\right)\right] .
$$

The large values of $\Delta_{11}^{(1)} \sim M_{Z}^{2}$ can be obtained only if $M_{S} \gg m_{t}$ and the ratio $\left|X_{t} / M_{S}\right|$ is also large. The contribution of the one-loop corrections (2.16) attains its maximal value for $X_{t}^{2}=6 M_{S}^{2}$. This is the so-called maximal mixing scenario. In Figs. $1 \mathrm{a}$ and $1 \mathrm{~b}$ the dependence of the one-loop lightest Higgs boson mass on the SUSY breaking scale $M_{S}$ for $\tan \beta=2$ and $\tan \beta=3$ is examined. Two different cases $X_{t}=M_{S}$ and $X_{t}=2 M_{S}$ are considered. From Figs. 1a and $1 \mathrm{~b}$ one can see that in order to satisfy LEP constraints $M_{S}$ should be larger than $400-600 \mathrm{GeV}$. Leading two-loop corrections reduce the SM-like Higgs mass even further. As a result larger values of the SUSY breaking scale are required to overcome LEP limit. 
Large values of the soft scalar masses of the superpartners of the top quark $\left(m_{Q}^{2}, m_{U}^{2} \gg M_{Z}^{2}\right)$ tend to induce large mass parameter $m_{2}^{2}$ at low energies due to the RG flow. This leads to the fine tuning because $m_{1}^{2}$ and $m_{2}^{2}$ determine the EW scale (see Eqs. (2.6)). Generically the fine tuning which is required to overcome LEP constraints in the MSSM is of the order of 1\% (little hierarchy problem). This fine tuning should be compared with the fine tuning in other theories which are used in particle physics. In particular, it is well known that QCD is a highly fine-tuned theory. Indeed, QCD Lagrangian should contain " $\theta$-term"

$$
\mathscr{L}_{\theta}=\theta_{\mathrm{eff}} \frac{\alpha_{s}}{8 \pi} F^{\mu v a} \tilde{F}_{\mu \nu}^{a}, \quad \theta_{\mathrm{eff}}=\theta+\arg \operatorname{det} M_{q},
$$

where $F^{\mu v a}$ is the gluon field strength and $\tilde{F}_{\mu \nu}^{a} \equiv \frac{1}{2} \varepsilon_{\mu v \rho \sigma} F^{\rho \sigma a}$ is its dual. This term is not forbidden by the gauge invariance. On the other hand the parameter $\theta$ must be extremely small, i.e. $\left|\theta_{\text {eff }}\right| \lesssim$ $10^{-9}$ (strong CP problem). Otherwise it results in too large value of the neutron electric dipole moment. Eqs. (2.17) demonstrate that so small value of $\theta_{\text {eff }}$ implies enormous fine tuning which is much higher than in the MSSM.

The little hierarchy problem can be solved within SUSY models that allow to get relatively large mass of the SM-like Higgs boson $\left(m_{h_{1}} \gtrsim 100-110 \mathrm{GeV}\right)$ at the tree-level. Alternatively, one can try to avoid stringent LEP constraints by allowing exotic decays of the lightest Higgs particle. If usual branching ratios of the lightest Higgs state are dramatically reduced then the lower LEP bound on the SM-like Higgs mass may become inapplicable. In this case the lightest Higgs boson can be still relatively light so that large contribution of loop corrections is not required. Both possibilities mentioned above imply the presence of new particles and interactions. These new particles and interactions can be also used to solve the so-called $\mu$ problem. This problem arises when MSSM gets incorporated into supergravity and/or GUT models. Within these models the parameter $\mu$ is expected to be either zero or of the order of Planck/GUT scale. At the same time in order to provide the correct pattern of the EWSB $\mu$ is required to be of the order of the EW scale.

\section{Higgs sector of the NMSSM}

In the simplest extension of the MSSM, the Next-to-Minimal Supersymmetric Standard Model (NMSSM), the superpotential is invariant with respect to the discrete transformations $\phi_{\alpha}^{\prime} \rightarrow e^{2 \pi i / 3} \phi_{\alpha}$ of the $Z_{3}$ group (for recent review see [2]). The term $\mu\left(H_{1} H_{2}\right)$ does not meet this requirement. Therefore it is replaced in the superpotential by

$$
W_{H}=\lambda S\left(H_{1} H_{2}\right)+\frac{1}{3} \kappa S^{3},
$$

where $S$ is an additional superfield which is a singlet with respect to $S U(2)_{W}$ and $U(1)_{Y}$ gauge transformations. A spontaneous breakdown of the EW symmetry leads to the emergence of the VEV of extra singlet field $\langle S\rangle=s / \sqrt{2}$ and an effective $\mu$ parameter is generated $(\mu=\lambda s / \sqrt{2})$.

The potential energy of the Higgs field interaction can be written as a sum

$$
\begin{gathered}
V=V_{F}+V_{D}+V_{\text {soft }}+\Delta V, \\
V_{F}=\lambda^{2}|S|^{2}\left(\left|H_{1}\right|^{2}+\left|H_{2}\right|^{2}\right)+\lambda^{2}\left|\left(H_{1} H_{2}\right)\right|^{2}+\lambda \kappa\left[S^{* 2}\left(H_{1} H_{2}\right)+\text { h.c. }\right]+\kappa^{2}|S|^{4},
\end{gathered}
$$




$$
\begin{gathered}
V_{D}=\frac{g_{2}^{2}}{8}\left(H_{1}^{+} \sigma_{a} H_{1}+H_{2}^{+} \sigma_{a} H_{2}\right)^{2}+\frac{g^{\prime 2}}{8}\left(\left|H_{1}\right|^{2}-\left|H_{2}\right|^{2}\right)^{2}, \\
V_{\text {soft }}=m_{1}^{2}\left|H_{1}\right|^{2}+m_{2}^{2}\left|H_{2}\right|^{2}+m_{S}^{2}|S|^{2}+\left[\lambda A_{\lambda} S\left(H_{1} H_{2}\right)+\frac{\kappa}{3} A_{\kappa} S^{3}+\text { h.c. }\right] .
\end{gathered}
$$

At the tree level the Higgs potential (3.2) is described by the sum of the first three terms. $V_{F}$ and $V_{D}$ are the $F$ and $D$ terms. Their structure is fixed by the superpotential (3.1) and the EW gauge interactions in the common manner. The soft SUSY breaking terms are collected in $V_{\text {soft }}$. The set of soft SUSY breaking parameters involves soft masses $m_{1}^{2}, m_{2}^{2}, m_{S}^{2}$ and trilinear couplings $A_{\kappa}, A_{\lambda}$. The last term in Eq. (3.2), $\Delta V$, corresponds to the contribution of loop corrections. In the leading one-loop approximation $\Delta V$ in the NMSSM is given by Eqs. (2.8) in which $\mu$ has to be replaced by $\lambda s / \sqrt{2}$. Further we assume that $\lambda, \kappa$ and all soft SUSY breaking parameters are real so that CP is conserved.

At the physical vacuum of the Higgs potential

$$
<H_{1}>=\frac{1}{\sqrt{2}}\left(\begin{array}{c}
v_{1} \\
0
\end{array}\right), \quad<H_{2}>=\frac{1}{\sqrt{2}}\left(\begin{array}{c}
0 \\
v_{2}
\end{array}\right), \quad<S>=\frac{s}{\sqrt{2}} .
$$

The equations for the extrema of the full Higgs boson effective potential in the directions (3.6) in the field space are given by

$$
\begin{aligned}
& \frac{\partial V}{\partial s}=\left(m_{S}^{2}+\frac{\lambda^{2}}{2}\left(v_{1}^{2}+v_{2}^{2}\right)-\lambda \kappa v_{1} v_{2}\right) s-\frac{\lambda A_{\lambda}}{\sqrt{2}} v_{1} v_{2}+\frac{\kappa A_{\kappa}}{\sqrt{2}} s^{2}+\kappa^{2} s^{3}+\frac{\partial \Delta V}{\partial s}=0 \\
& \frac{\partial V}{\partial v_{1}}=\left(m_{1}^{2}+\frac{\lambda^{2}}{2}\left(v_{2}^{2}+s^{2}\right)+\frac{\bar{g}^{2}}{8}\left(v_{1}^{2}-v_{2}^{2}\right)\right) v_{1}-\left(\frac{\lambda \kappa}{2} s^{2}+\frac{\lambda A_{\lambda}}{\sqrt{2}} s\right) v_{2}+\frac{\partial \Delta V}{\partial v_{1}}=0, \\
& \frac{\partial V}{\partial v_{2}}=\left(m_{2}^{2}+\frac{\lambda^{2}}{2}\left(v_{1}^{2}+s^{2}\right)+\frac{\bar{g}^{2}}{8}\left(v_{2}^{2}-v_{1}^{2}\right)\right) v_{2}-\left(\frac{\lambda \kappa}{2} s^{2}+\frac{\lambda A_{\lambda}}{\sqrt{2}} s\right) v_{1}+\frac{\partial \Delta V}{\partial v_{2}}=0 .
\end{aligned}
$$

As in the MSSM upon the breakdown of the EW symmetry three goldstone modes $\left(G^{ \pm}\right.$and $\left.G^{0}\right)$ emerge, and are absorbed by the $W^{ \pm}$and $Z$ bosons. In the field space basis rotated by an angle $\beta$ with respect to the initial direction, i.e.

$$
\begin{array}{ll}
H_{1}^{-}=G^{-} \cos \beta+H^{-} \sin \beta, & H_{2}^{+}=H^{+} \cos \beta-G^{+} \sin \beta, \\
\operatorname{Im} H_{1}^{0}=\left(P \sin \beta+G^{0} \cos \beta\right) / \sqrt{2}, & \operatorname{Re} H_{1}^{0}=\left(h \cos \beta-H \sin \beta+v_{1}\right) / \sqrt{2}, \\
\operatorname{Im} H_{2}^{0}=\left(P \cos \beta-G^{0} \sin \beta\right) / \sqrt{2}, & \operatorname{Re} H_{2}^{0}=\left(h \sin \beta+H \cos \beta+v_{2}\right) / \sqrt{2}, \\
\operatorname{Im} S=P_{S} / \sqrt{2}, & \operatorname{Re} S=(s+N) / \sqrt{2},
\end{array}
$$

these unphysical degrees of freedom decouple and the mass terms in the Higgs boson potential can be written as follows

$$
V_{\text {mass }}=M_{H^{ \pm}}^{2} H^{+} H^{-}+\frac{1}{2}\left(P P_{S}\right) \tilde{M}^{2}\left(\begin{array}{c}
P \\
P_{S}
\end{array}\right)+\frac{1}{2}(H h S) M^{2}\left(\begin{array}{c}
H \\
h \\
S
\end{array}\right) .
$$

From the conditions for the extrema (3.7)-(3.9) one can express $m_{S}^{2}, m_{1}^{2}, m_{2}^{2}$ via other fundamental parameters, $\tan \beta$ and $s$. Substituting the obtained relations for the soft masses in the $2 \times 2$ CP-odd mass matrix $\tilde{M}_{i j}^{2}$ we get:

$$
\tilde{M}_{11}^{2}=m_{A}^{2}=\frac{4 \mu^{2}}{\sin ^{2} 2 \beta}\left(x-\frac{\kappa}{2 \lambda} \sin 2 \beta\right)+\tilde{\Delta}_{11}, \quad \tilde{M}_{22}^{2}=\frac{\lambda^{2} v^{2}}{2} x+\frac{\lambda \kappa}{2} v^{2} \sin 2 \beta-3 \frac{\kappa}{\lambda} A_{\kappa} \mu+\tilde{\Delta}_{22},
$$




$$
\tilde{M}_{12}^{2}=\tilde{M}_{21}^{2}=\sqrt{2} \lambda v \mu\left(\frac{x}{\sin 2 \beta}-2 \frac{\kappa}{\lambda}\right)+\tilde{\Delta}_{12},
$$

where $x=\frac{1}{2 \mu}\left(A_{\lambda}+2 \frac{\kappa}{\lambda} \mu\right) \sin 2 \beta, \mu=\frac{\lambda s}{\sqrt{2}}$ and $\tilde{\Delta}_{i j}$ are contributions of the loop corrections to the mass matrix elements. The mass matrix (3.12) can be easily diagonalized. The corresponding eigenvalues are given by

$$
m_{A_{2}, A_{1}}^{2}=\frac{1}{2}\left(\tilde{M}_{11}^{2}+\tilde{M}_{22}^{2} \pm \sqrt{\left(\tilde{M}_{11}^{2}-\tilde{M}_{22}^{2}\right)^{2}+4 \tilde{M}_{12}^{4}}\right)
$$

Because the charged components of the Higgs doublets are not mixed with the neutral Higgs states the charged Higgs fields $H^{ \pm}$are already physical mass eigenstates with

$$
M_{H^{ \pm}}^{2}=m_{A}^{2}-\frac{\lambda^{2} v^{2}}{2}+M_{W}^{2}+\Delta_{ \pm}
$$

Here $\Delta_{ \pm}$includes loop corrections to the charged Higgs mass.

In the rotated basis $H, h, N$ the mass matrix of the CP-even Higgs sector takes the form [1], [3]:

$$
\begin{aligned}
M^{2}=\left(\begin{array}{lll}
M_{11}^{2} & M_{12}^{2} & M_{13}^{2} \\
M_{21}^{2} & M_{22}^{2} & M_{23}^{2} \\
M_{31}^{2} & M_{32}^{2} & M_{33}^{2}
\end{array}\right)=\left(\begin{array}{ccc}
\frac{1}{v^{2}} \frac{\partial^{2} V}{\partial^{2} \beta} & \frac{1}{v} \frac{\partial^{2} V}{\partial v \partial \beta} & \frac{1}{v} \frac{\partial^{2} V}{\partial s \partial \beta} \\
\frac{1}{v} \frac{\partial^{2} V}{\partial v \partial \beta} & \frac{\partial^{2} V}{\partial v^{2}} & \frac{\partial^{2} V}{\partial v \partial s} \\
\frac{1}{v} \frac{\partial^{2} V}{\partial s \partial \beta} & \frac{\partial^{2} V}{\partial v \partial s} & \frac{\partial^{2} V}{\partial^{2} s}
\end{array}\right), \\
M_{11}^{2}=m_{A}^{2}+\left(\frac{\bar{g}^{2}}{4}-\frac{\lambda^{2}}{2}\right) v^{2} \sin ^{2} 2 \beta+\Delta_{11}, \\
M_{22}^{2}=M_{Z}^{2} \cos ^{2} 2 \beta+\frac{\lambda^{2}}{2} v^{2} \sin ^{2} 2 \beta+\Delta_{22}, \\
M_{33}^{2}=4 \frac{\kappa^{2}}{\lambda^{2}} \mu^{2}+\frac{\kappa}{\lambda} A_{\kappa} \mu+\frac{\lambda^{2} v^{2}}{2} x-\frac{\kappa \lambda}{2} v^{2} \sin 2 \beta+\Delta_{33}, \\
M_{12}^{2}=M_{21}^{2}=\left(\frac{\lambda^{2}}{4}-\frac{\bar{g}^{2}}{8}\right) v^{2} \sin 4 \beta+\Delta_{12} \\
M_{13}^{2}=M_{31}^{2}=-\sqrt{2} \lambda v \mu x \operatorname{ctg} 2 \beta+\Delta_{13}, \\
M_{23}^{2}=M_{32}^{2}=\sqrt{2} \lambda v \mu(1-x)+\Delta_{23},
\end{aligned}
$$

where $\Delta_{i j}$ can be calculated by differentiating $\Delta V$.

At least one Higgs state in the $\mathrm{CP}$-even sector is always light. Since the minimal eigenvalue of a Hermitian matrix does not exceed its smallest diagonal element the lightest $\mathrm{CP}$-even Higgs boson squared mass $m_{h_{1}}^{2}$ remains smaller than $M_{22}^{2} \sim M_{Z}^{2}$ even when the supersymmetry breaking scale is much larger than the EW scale ${ }^{1}$. The upper bound on the lightest Higgs mass in the NMSSM differs from the theoretical bound in the MSSM only for moderate values of $\tan \beta$. In the leading two-loop approximation the lightest Higgs boson mass in the NMSSM does not exceed $135 \mathrm{GeV}$. As follows from the explicit form of the mass matrices (3.12) and (3.16) at the tree-level, the spectrum of the Higgs bosons and their couplings depend on the six parameters: $\lambda, \kappa, \mu, \tan \beta, A_{\kappa}$ and $m_{A}$ (or $x$ ).

\footnotetext{
${ }^{1}$ The same theorem may lead to the upper bound on the mass of the lightest neutralino [4].
} 
First let us consider the MSSM limit of the NMSSM. Because the strength of the interaction of the extra SM singlet superfield $S$ with $H_{1}$ and $H_{2}$ is determined by the size of the coupling $\lambda$ in the superpotential (3.1) the MSSM expressions for the Higgs masses and couplings are reproduced when $\lambda$ tends to be zero. On the other hand the equations (3.8)-(3.9) imply that $s$ should grow with decreasing $\lambda$ as $M_{Z} / \lambda$ to ensure the correct breakdown of the EW symmetry. In the limit $\lambda \rightarrow 0$ all terms, which are proportional to $\lambda v_{i}$, in the minimization conditions (3.7) can be neglected and the corresponding equation takes the form:

$$
s\left(m_{S}^{2}+\frac{\kappa A_{\kappa}}{\sqrt{2}} s+\kappa^{2} s^{2}\right) \simeq 0
$$

The Eq. (3.17) has always at least one solution $s_{0}=0$. In addition two non-trivial roots arise if $A_{\kappa}^{2}>8 m_{S}^{2}$. They are given by

$$
s_{1,2} \simeq \frac{-A_{\kappa} \pm \sqrt{A_{\kappa}^{2}-8 m_{S}^{2}}}{2 \sqrt{2} \kappa} .
$$

When $m_{S}^{2}>0$ the root $s_{0}=0$ corresponds to the local minimum of the Higgs potential (3.2) $-(3.5)$ that does not lead to the acceptable solution of the $\mu$-problem. The second non-trivial vacuum, that appears if $A_{\kappa}^{2}>8 m_{S}^{2}$, remains unstable for $A_{\kappa}^{2}<9 m_{S}^{2}$. Larger absolute values of $A_{\kappa}\left(A_{\kappa}^{2}>9 m_{S}^{2}\right)$ stabilizes the second minimum which is attained at $s=s_{1}\left(s_{2}\right)$ for negative (positive) $A_{\kappa}$. From Eq. (3.18) it becomes clear that the increasing of $s$ can be achieved either by decreasing $\kappa$ or by raising $m_{S}^{2}$ and $A_{\kappa}$. Since there is no natural reason why $m_{S}^{2}$ and $A_{\kappa}$ should be very large while all other soft SUSY breaking terms are left in the TeV range, the values of $\lambda$ and $\kappa$ are obliged to go to zero simultaneously so that their ratio remains unchanged.

Since in the MSSM limit of the NMSSM mixing between singlet states and neutral components of the Higgs doublets is small the mass matrices (3.12) and (3.15)-3.16) can be diagonalised

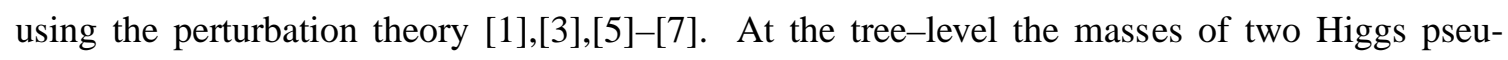
doscalars are given by

$$
m_{A_{2}}^{2} \simeq m_{A}^{2}=\frac{4 \mu^{2}}{\sin ^{2} 2 \beta}\left(x-\frac{\kappa}{2 \lambda} \sin 2 \beta\right), \quad m_{A_{1}}^{2} \simeq-3 \frac{\kappa}{\lambda} A_{\kappa} \mu .
$$

The masses of two CP-even Higgs bosons are the same as in the MSSM (see Eq. (2.12)) while the mass of the extra CP-even Higgs state, which is predominantly a SM singlet field, is set by $\frac{\kappa}{\lambda} \mu$

$$
m_{h_{3}}^{2} \approx 4 \frac{\kappa^{2}}{\lambda^{2}} \mu^{2}+\frac{\kappa}{\lambda} A_{\kappa} \mu+\frac{\lambda^{2} v^{2}}{2} x \sin ^{2} 2 \beta-\frac{2 \lambda^{2} v^{2} \mu^{2}(1-x)^{2}}{M_{Z}^{2} \cos ^{2} 2 \beta} .
$$

The parameter $A_{\kappa}$ occurs in the masses of extra scalar $m_{h_{3}}$ and pseudoscalar $m_{A_{1}}$ with opposite sign and is therefore responsible for their splitting. To ensure that the physical vacuum is a global minimum of the Higgs potential (3.2)-(3.5) and the masses-squared of all Higgs states are positive in this vacuum the parameter $A_{\kappa}$ must satisfy the following constraints

$$
-3\left(\frac{\kappa}{\lambda} \mu\right)^{2} \lesssim A_{\kappa}\left(\frac{\kappa}{\lambda} \mu\right) \lesssim 0
$$

The experimental constraints on the SUSY parameters obtained in the MSSM remain valid in the NMSSM with small $\lambda$ and $\kappa$. For example, non-observation of any neutral Higgs particle and chargino at the LEP II imply that $\tan \beta \gtrsim 2.5$ and $|\mu| \gtrsim 90-100 \mathrm{GeV}$. 


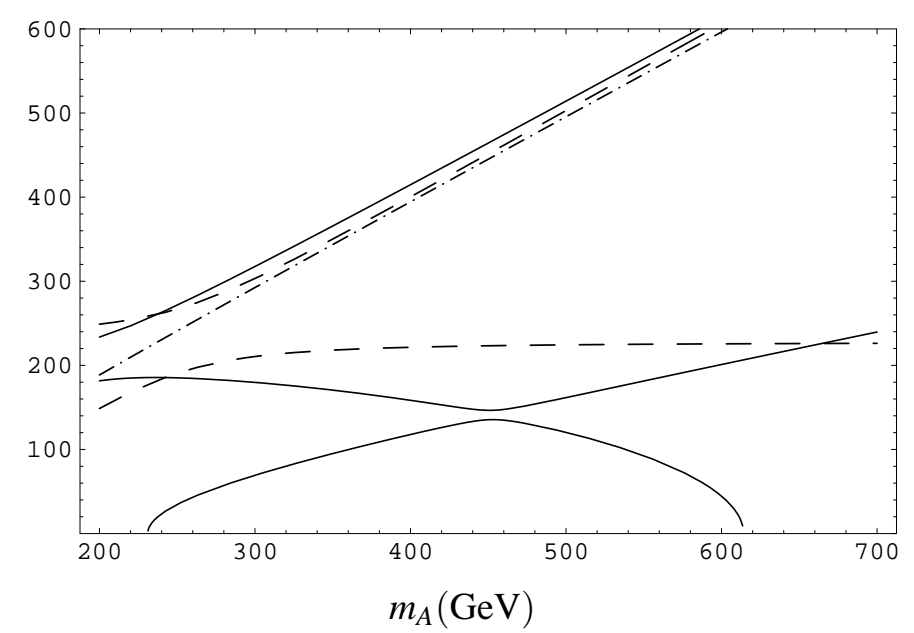

Figure 2: One-loop masses of the CP-even Higgs bosons versus $m_{A}$ for $\lambda=0.6, \kappa=0.36, \tan \beta=3$, $\mu=150 \mathrm{GeV}, A_{\kappa}=135 \mathrm{GeV}, m_{Q}^{2}=m_{U}^{2}=M_{S}^{2}, X_{t}=\sqrt{6} M_{S}$ and $M_{S}=700 \mathrm{GeV}$. Solid, dashed and dasheddotted lines correspond to the masses of the $\mathrm{CP}-$ even, $\mathrm{CP}$-odd and charged Higgs bosons respectively.

Decreasing $\kappa$ reduces the masses of extra scalar and pseudoscalar states so that for $\kappa \ll \lambda$ they can be the lightest particles in the Higgs boson spectrum. In the limit $\kappa \rightarrow 0$ the mass of the lightest pseudoscalar state vanishes. In the considered limit the Lagrangian of the NMSSM is invariant under transformations of $S U(2) \times[U(1)]^{2}$ global symmetry. Extra $U(1)$ global symmetry gets spontaneously broken by the VEV of the singlet field $S$, giving rise to a massless Goldstone boson, the Peccei-Quinn (PQ) axion. In the PQ-symmetric NMSSM astrophysical observations exclude any choice of the parameters unless one allows $s$ to be enormously large $\left(>10^{9}-10^{11} \mathrm{GeV}\right)$. These huge vacuum expectation values of the singlet field can be consistent with the EWSB only if $\lambda \sim 10^{-6}-10^{-9}$ [8]-[9]. Therefore here we restrict our consideration to small but non-zero values of $\kappa \lesssim \lambda^{2}$ when the PQ-symmetry is only slightly broken.

As evident from Eq. (3.20) at small values of $\kappa$ the mass-squared of the lightest Higgs scalar tends to be negative if $|\mu|$ is large and/or the auxiliary variable $x$ differs too much from unity. Due to the vacuum stability requirement, which implies the positivity of the mass-squared of all Higgs particles, $x$ has to be localized near unity, i.e.

$$
1-\left|\frac{\sqrt{2} \kappa M_{Z}}{\lambda^{2} v}\right|<x<1+\left|\frac{\sqrt{2} \kappa M_{Z}}{\lambda^{2} v}\right| .
$$

This leads to the hierarchical structure of the Higgs spectrum. Indeed, combining LEP limits on $\tan \beta$ and $\mu$ one gets that $m_{A}^{2} \gtrsim 9 M_{Z}^{2} x$. Because of this the heaviest CP-odd, CP-even and charged Higgs bosons are almost degenerate with masses around $m_{A} \simeq \mu \tan \beta$ while the SM-like Higgs state has a mass of the order of $M_{Z}$.

The main features of the NMSSM Higgs spectrum discussed above are retained when the couplings $\lambda$ and $\kappa$ increase. For the appreciable values of $\kappa$ and $\lambda$ the slight breaking of the PQsymmetry can be caused by the RG flow of these couplings from the GUT scale $M_{X}$ to $M_{Z}$. In the infrared region the solutions of the NMSSM RG equations are focused near the intersection of the Hill-type effective surface and invariant line [10]-[12]. As a result at the EW scale $\kappa / \lambda$ tend to be 
less than unity even when $\kappa\left(M_{X}\right)>\lambda\left(M_{X}\right)$ initially. In Fig. 2 the dependence of the masses and couplings of the Higgs bosons on $m_{A}$ is examined. As a representative example we fix the Yukawa couplings so that $\lambda\left(M_{X}\right)=\kappa\left(M_{X}\right)=2 h_{t}\left(M_{X}\right)=1.6$, that corresponds to $\tan \beta \gtrsim 3, \lambda\left(M_{t}\right)=0.6$ and $\kappa\left(M_{t}\right)=0.36$. In order to obtain a realistic spectrum, we include the leading one-loop corrections from the top and stop loops. From Fig. 2 it becomes clear that the requirement of stability of the physical vacuum limits the range of variations of $m_{A}$ from below and above maintaining the mass hierarchy in the Higgs spectrum. Relying on this mass hierarchy the approximate solutions for the Higgs masses and couplings can be obtained [6], [7]. The numerical results in Fig. 2 reveal that the masses of the heaviest $\mathrm{CP}-$ even, $\mathrm{CP}$-odd and charged Higgs states are approximately degenerate while the other three neutral states are considerably lighter. The hierarchical structure of the Higgs spectrum ensures that the heaviest $\mathrm{CP}$-even and $\mathrm{CP}$-odd Higgs bosons are predominantly composed of $H$ and $P$. As before the lightest Higgs scalar and pseudoscalar are singlet dominated, making their observation quite problematic. The second lightest $\mathrm{CP}$-even Higgs boson has a mass around $130 \mathrm{GeV}$, mimicking the lightest Higgs scalar in the MSSM. Observing two light scalars and one pseudoscalar Higgs particles but no charged Higgs boson at future colliders would yield an opportunity to differentiate the NMSSM with a slightly broken PQ-symmetry from the MSSM even if the heavy Higgs states are inaccessible.

The presence of light singlet scalar and pseudoscalar permits to weaken the LEP lower bound on the lightest Higgs boson mass. These states have reduced couplings to Z-boson that could allow them to escape the detection at LEP. On the other hand singlet scalar can mix with the SMlike superposition $h$ of the neutral components of Higgs doublets resulting in the reduction of the couplings of the second lightest Higgs scalar to Z-boson. This relaxes LEP constraints so that the SM-like Higgs state does not need to be considerably heavier than $100 \mathrm{GeV}$. Therefore large contribution of loop corrections to the mass of the SM-like Higgs boson is not required. Another possibility to overcome the little hierarchy problem is to allow the SM-like Higgs state to decay predominantly into two light singlet pseudoscalars $A_{1}$ (for recent review see [13]). This can be achieved because the coupling of the SM-like Higgs boson to the $b$-quark is rather small. If this coupling is substantially smaller than the coupling of the SM-like Higgs state to $A_{1}$ then the decay mode $h \rightarrow A_{1}+A_{1}$ dominates. The singlet pseudoscalar can sequentially decay into either $b \bar{b}$ or $\tau \bar{\tau}$ leading to four fermion decays of the SM-like Higgs boson. In this case, again, the corresponding Higgs eigenstate might be relatively light that permits to avoid little hierarchy problem.

However even when the couplings of the lightest CP-even Higgs state are almost the same as in the SM it is substantially easier to overcome LEP constraint on the mass of the SM-like Higgs boson in the NMSSM than in the MSSM. Indeed, in the NMSSM the theoretical upper bound on $m_{h_{1}}^{2}$, which is given by $M_{22}^{2}$ in Eq. (3.16), contains an extra term $\frac{\lambda^{2}}{2} v^{2} \sin ^{2} 2 \beta$ which is not present in the MSSM. Due to this term the maximum possible value of the mass of the lightest Higgs scalar in the NMSSM can be considerably larger as compared with the MSSM at moderate values of $\tan \beta$. In our analysis we require the validity of perturbation theory up to the scale $M_{X}$. This sets stringent upper limit on $\lambda\left(M_{t}\right)$ at low energies for each particular choice of $\tan \beta$. Using theoretical restrictions on $\lambda\left(M_{t}\right)$ one can compute the the maximum possible value of $m_{h_{1}}^{2}$ for each given value of $\tan \beta$. Fig. 3 shows the dependence of the upper bound on the lightest Higgs boson mass as a function of $\tan \beta$ in the MSSM and NMSSM. From Fig. 3 one can see that at the tree-level the lightest CP-even Higgs state in the NMSSM can be considerably heavier than in the 

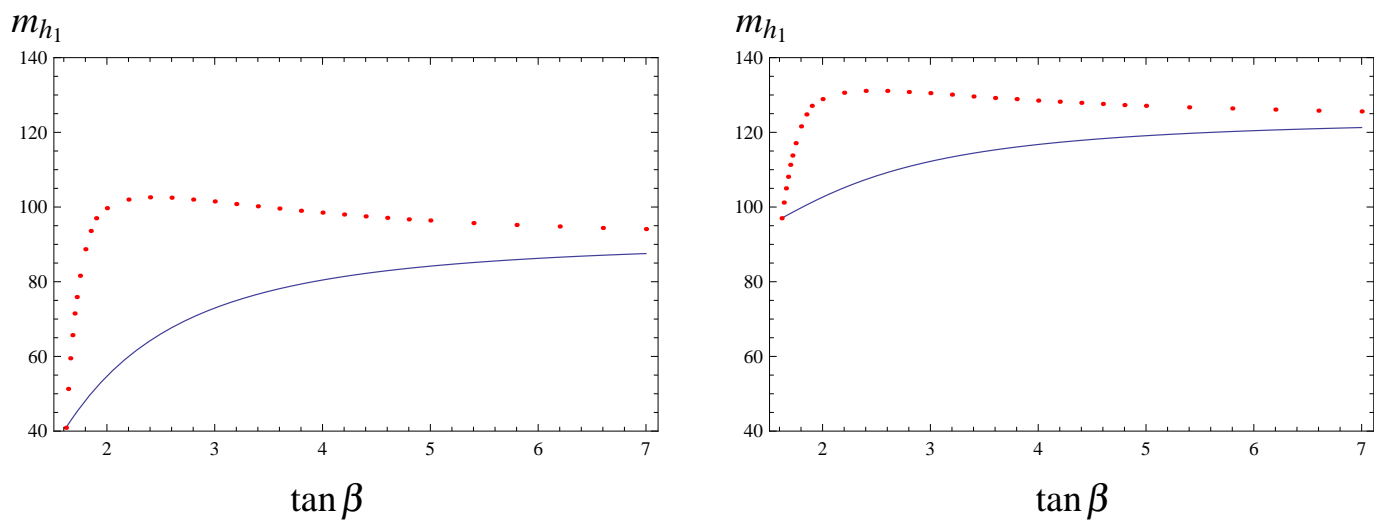

Figure 3: Left: Tree-level upper bound on the lightest Higgs boson mass in the MSSM and NMSSM as a function of $\tan \beta$. Right: The dependence of the two-loop upper bound on the lightest Higgs boson mass on $\tan \beta$ for $m_{t}\left(M_{t}\right)=165 \mathrm{GeV}, m_{Q}^{2}=m_{U}^{2}=M_{S}^{2}, X_{t}=\sqrt{6} M_{S}$ and $M_{S}=700 \mathrm{GeV}$. The solid and dotted lines represent the theoretical restrictions on $m_{h_{1}}$ in the MSSM and NMSSM respectively.

MSSM at moderate values of $\tan \beta$. As a consequence in the leading two-loop approximation it is substantially easier to get $m_{h_{1}} \gtrsim 114.4 \mathrm{GeV}$ in the NMSSM than in the MSSM for $\tan \beta=2-4$.

\section{Higgs spectrum in the $E_{6}$ inspired SUSY models with extra $U(1)^{\prime}$ factor}

Another solution to the $\mu$ problem arises within superstring inspired models based on the $E_{6}$ gauge group. At high energies $E_{6}$ can be broken $S U(3)_{C} \times S U(2)_{W} \times U(1)_{Y} \times U(1)^{\prime}$. An extra $U(1)^{\prime}$ that appears at low energies is a linear superposition of $U(1)_{\chi}$ and $U(1)_{\psi}$ :

$$
U(1)^{\prime}=U(1)_{\chi} \cos \theta+U(1)_{\psi} \sin \theta,
$$

where two anomaly-free $U(1)_{\psi}$ and $U(1)_{\chi}$ symmetries are defined by: $E_{6} \rightarrow S O(10) \times U(1)_{\psi}$, $S O(10) \rightarrow S U(5) \times U(1)_{\chi}$. If $\theta \neq 0$ or $\pi$ the extra $U(1)^{\prime}$ gauge symmetry forbids an elementary $\mu$ term but allows interaction $\lambda S\left(H_{1} H_{2}\right)$ in the superpotential. After EWSB the scalar component of the SM singlet superfield $S$ acquires a non-zero VEV breaking $U(1)^{\prime}$ and an effective $\mu$ term of the required size is automatically generated.

The Higgs sector of the considered models includes two Higgs doublets as well as a SM-like singlet field $S$ that carries $U(1)^{\prime}$ charge. The Higgs effective potential can be written as

$$
\begin{aligned}
V= & V_{F}+V_{D}+V_{\text {soft }}+\Delta V, \\
V_{F}= & \lambda^{2}|S|^{2}\left(\left|H_{d}\right|^{2}+\left|H_{u}\right|^{2}\right)+\lambda^{2}\left|\left(H_{d} H_{u}\right)\right|^{2}, \\
V_{D}= & \frac{g_{2}^{2}}{8}\left(H_{d}^{\dagger} \sigma_{a} H_{d}+H_{u}^{\dagger} \sigma_{a} H_{u}\right)^{2}+\frac{g^{\prime 2}}{8}\left(\left|H_{d}\right|^{2}-\left|H_{u}\right|^{2}\right)^{2} \\
& +\frac{g_{1}^{\prime 2}}{2}\left(\tilde{Q}_{1}\left|H_{d}\right|^{2}+\tilde{Q}_{2}\left|H_{u}\right|^{2}+\tilde{Q}_{S}|S|^{2}\right)^{2}, \\
V_{\text {soft }}= & m_{S}^{2}|S|^{2}+m_{1}^{2}\left|H_{d}\right|^{2}+m_{2}^{2}\left|H_{u}\right|^{2}+\left[\lambda A_{\lambda} S\left(H_{u} H_{d}\right)+\text { h.c. }\right],
\end{aligned}
$$

where $g_{1}^{\prime}$ is $U(1)^{\prime}$ gauge coupling and $\tilde{Q}_{1}, \tilde{Q}_{2}$ and $\tilde{Q}_{S}$ are effective $U(1)^{\prime}$ charges of $H_{1}, H_{2}$ and $S$ respectively. In Eq. (4.2) $V_{F}$ and $V_{D}$ are the $F$ and $D$ terms, $V_{\text {soft }}$ contains a set of soft SUSY breaking terms while $\Delta V$ represents the contribution of loop corrections. 
At the physical vacuum the Higgs fields acquire VEVs given by Eq. (3.6) thus breaking the $S U(2)_{W} \times U(1)_{Y} \times U(1)^{\prime}$ symmetry to $U(1)_{e m}$. As a result two CP-odd and two charged Goldstone modes in the Higgs sector are absorbed by the $Z, Z^{\prime}$ and $W^{ \pm}$gauge bosons so that only six physical degrees of freedom are left. They form one $\mathrm{CP}-$ odd, three $\mathrm{CP}-$ even and two charged states. The masses of the $\mathrm{CP}-$ odd and charged Higgs bosons can be written as

$$
m_{A}^{2}=\frac{2 \lambda^{2} s^{2} x}{\sin ^{2} 2 \beta}+O\left(M_{Z}^{2}\right), \quad m_{H^{ \pm}}^{2}=m_{A}^{2}+O\left(M_{Z}^{2}\right)
$$

where $x=\frac{A_{\lambda}}{\sqrt{2} \lambda s} \sin 2 \beta$. The masses of two heaviest CP-even states are set by $M_{Z^{\prime}}$ and $m_{A}$, i.e.

$$
m_{h_{3}}^{2}=m_{A}^{2}+O\left(M_{Z}^{2}\right), \quad m_{h_{2}}^{2}=M_{Z^{\prime}}^{2}+O\left(M_{Z}^{2}\right)
$$

where $M_{Z^{\prime}} \simeq g_{1}^{\prime} \tilde{Q}_{S} s$. The lightest CP-even Higgs boson has a mass which is less than

$$
m_{h_{1}}^{2} \lesssim \frac{\lambda^{2}}{2} v^{2} \sin ^{2} 2 \beta+M_{Z}^{2} \cos ^{2} 2 \beta+g_{1}^{\prime 2} v^{2}\left(\tilde{Q}_{1} \cos ^{2} \beta+\tilde{Q}_{2} \sin ^{2} \beta\right)^{2}+\Delta .
$$

In Eq. (4.5) $\Delta$ represents the contribution of loop corrections. Since the mass of the $Z^{\prime}$ boson in the $E_{6}$ inspired models has to be heavier than $800-900 \mathrm{GeV}$ at least one CP-even Higgs state, which is singlet dominated, is always heavy. If $m_{A}<M_{Z^{\prime}}$ then we get MSSM-type Higgs spectrum. When $m_{A}>M_{Z^{\prime}}$ the heaviest $\mathrm{CP}$-even, $\mathrm{CP}$-odd and charged states are almost degenerate with masses around $m_{A}$. In this case the lightest Higgs state is predominantly the SM-like superposition $h$ of the neutral components of Higgs doublets.

Recently the detailed analysis of the Higgs sector was performed within a particular $E_{6}$ inspired SUSY model with an extra $U(1)_{N}$ gauge symmetry that corresponds to $\theta=\arctan \sqrt{15}$ [14]-[15]. The extra $U(1)_{N}$ gauge symmetry is defined such that right-handed neutrinos do not participate in the gauge interactions. Only in this Exceptional Supersymmetric Standard Model ( $\mathrm{E}_{6} \mathrm{SSM}$ ) right-handed may be superheavy, shedding light on the origin of the mass hierarchy in the lepton sector and providing a mechanism for the generation of the baryon asymmetry in the Universe via leptogenesis [16]. To ensure anomaly cancellation the particle content of the $\mathrm{E}_{6} \mathrm{SSM}$ is extended to include three complete fundamental 27 representations of $E_{6}$. In addition to the complete $27_{i}$ multiplets the low energy particle spectrum of the $\mathrm{E}_{6} \mathrm{SSM}$ is supplemented by $S U(2)_{W}$ doublet $H^{\prime}$ and anti-doublet $\bar{H}^{\prime}$ states from extra $27^{\prime}$ and $\overline{27^{\prime}}$ to preserve gauge coupling unification. The unification of gauge couplings in the considered model can be achieved for any phenomenologically acceptable value of $\alpha_{3}\left(M_{Z}\right)$ consistent with the measured low energy central value [17]. The Higgs spectrum within the $\mathrm{E}_{6} \mathrm{SSM}$ was studied in [14]-[15], [18]-[19]. It was argued that even at the tree level the lightest Higgs boson mass in this model can be larger than $120 \mathrm{GeV}$. Therefore nonobservation of the Higgs boson at LEP does not cause any trouble for the $\mathrm{E}_{6} \mathrm{SSM}$, even at tree-level. In the leading two-loop approximation the mass of the lightest $\mathrm{CP}-$ even Higgs boson in the considered model does not exceed $150-155 \mathrm{GeV}$ [14]. The presence of light exotic particles in the $\mathrm{E}_{6} \mathrm{SSM}$ spectrum lead to the nonstandard decays of the SM-like Higgs boson which were discussed in [20]. 


\section{References}

[1] P. A. Kovalenko, R. B. Nevzorov, K. A. Ter-Martirosian, Masses of Higgs bosons in supersymmetric theories, Phys. Atom. Nucl. 61 (1998) 812.

[2] U. Ellwanger, C. Hugonie, A. M. Teixeira, The Next-to-Minimal Supersymmetric Standard Model, Phys. Rept. 496 (2010) 1 [arXiv:0910.1785 [hep-ph]].

[3] R. B. Nevzorov, K. A. Ter-Martirosyan, M. A. Trusov, Higgs bosons in the simplest SUSY models, Phys. Atom. Nucl. 65 (2002) 285 [hep-ph/ 0105178$].$

[4] S. Hesselbach, D. J. Miller, G. Moortgat-Pick, R. Nevzorov, M. Trusov, Theoretical upper bound on the mass of the LSP in the MNSSM, Phys. Lett. B 662 (2008) 199 [arXiv: 0712.2001 [hep-ph]].

[5] R. B. Nevzorov, M. A. Trusov, Particle spectrum in the modified NMSSM in the strong Yukawa coupling limit, J. Exp. Theor. Phys. 91 (2000) 1079 [hep-ph/0106351];

[6] D. J. Miller, R. Nevzorov, P. M. Zerwas, The Higgs sector of the next-to-minimal supersymmetric standard model, Nucl. Phys. B 681 (2004) 3 [hep-ph/ 0304049 ].

[7] R. Nevzorov, D. J. Miller, Approximate solutions for the Higgs masses and couplings in the NMSSM, [hep-ph/0411275].

[8] D. J. Miller, R. Nevzorov, The Peccei-Quinn axion in the next-to-minimal supersymmetric standard model, [hep-ph/0309143].

[9] D. J. Miller, S. Moretti, R. Nevzorov, Higgs bosons in the NMSSM with exact and slightly broken $P Q$-symmetry, [hep-ph/0501139].

[10] R. B. Nevzorov, M. A. Trusov, Infrared quasi-fixed solutions in the NMSSM, Phys. Atom. Nucl. 64 (2001) 1299 [hep-ph/0110363].

[11] R. B. Nevzorov, M. A. Trusov, Renormalization of the soft SUSY breaking terms in the strong Yukawa coupling limit in the NMSSM, Phys. Atom. Nucl. 64 (2001) 1513 [hep-ph/0112301].

[12] R. B. Nevzorov, M. A. Trusov, Quasi-fixed point scenario in the modified NMSSM, Phys. Atom. Nucl. 65 (2002) 335 [hep-ph/ 0301179 ].

[13] S. Chang, R. Dermisek, J. F. Gunion and N. Weiner, Nonstandard Higgs Boson Decays, Ann. Rev. Nucl. Part. Sci. 58 (2008) 75 [arXiv:0801.4554 [hep-ph] ];

[14] S. F. King, S. Moretti, R. Nevzorov, Theory and phenomenology of an exceptional supersymmetric standard model, Phys. Rev. D 73 (2006) 035009 [hep-ph / 0510419 ].

[15] S. F. King, S. Moretti, R. Nevzorov, Exceptional supersymmetric standard model, Phys. Lett. B 634 (2006) 278 [hep-ph/0511256].

[16] S. F. King, R. Luo, D. J. . Miller and R. Nevzorov, Leptogenesis in the Exceptional Supersymmetric Standard Model: flavour dependent lepton asymmetries, JHEP 0812 (2008) 042 [arXiv:0806.0330 [hep-ph]].

[17] S. F. King, S. Moretti, R. Nevzorov, Gauge Coupling Unification in the Exceptional Supersymmetric Standard Model, Phys. Lett. B 650 (2007) 57 [hep-ph/ 0701064 ].

[18] S. F. King, S. Moretti, R. Nevzorov, Spectrum of Higgs particles in the ESSM, [hep-ph/0601269].

[19] S. F. King, S. Moretti, R. Nevzorov, E6SSM, AIP Conf. Proc. 881 (2007) 138 [hep-ph/ 0610002 ].

[20] J. P. Hall, S. F. King, R. Nevzorov, S. Pakvasa, M. Sher, Novel Higgs Decays and Dark Matter in the $E_{6} S S M,[\operatorname{arXiv}: 1012.5114$ [hep-ph]]. 